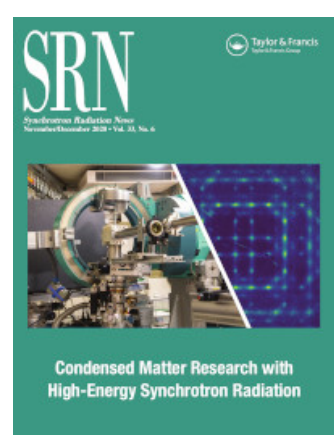

\title{
Synchrotron Radiation News
}

\section{Examples of High-energy X-ray Structural Studies at PETRA III: High $T_{\mathrm{C}}$ Superconductors, Real-time Surface and Thin Film Processes, and Design of Engineering Materials}

Ann-Christin Dippel, Martin V. Zimmermann, Peter Staron \& Jochen R. Schneider

To cite this article: Ann-Christin Dippel, Martin V. Zimmermann, Peter Staron \& Jochen R. Schneider (2020) Examples of High-energy X-ray Structural Studies at PETRA III: High $T_{\mathrm{C}}$ Superconductors, Real-time Surface and Thin Film Processes, and Design of Engineering Materials, Synchrotron Radiation News, 33:6, 24-30, DOI: 10.1080/08940886.2020.1841493

To link to this article: https://doi.org/10.1080/08940886.2020.1841493

曲 Published online: 28 Jan 2021.

Submit your article to this journal $\widetilde{ }$

\section{Article views: 83}

Q View related articles $₫$

View Crossmark data $\nearrow$ 


\title{
Examples of High-energy X-ray Structural Studies at PETRA III: High $T_{\mathrm{C}}$ Superconductors, Real-time Surface and Thin Film Processes, and Design of Engineering Materials
}

\author{
Ann-Christin Dippel, ${ }^{1}$ Martin V. Zimmermann, ${ }^{1}$ Peter Staron, $^{2}$ and Jochen R. Schneider ${ }^{1}$ \\ ${ }^{1}$ Deutsches Elektronen-Synchrotron DESY, Hamburg, Germany \\ ${ }^{2}$ Institute of Materials Research, Helmholtz-Zentrum Geesthacht, Geesthacht, Germany
}

\section{High-energy X-ray activities at DESY}

At DESY, systematic work with very hard X-rays started with the availability of the HARWI beamline at the DORIS storage ring in 1986 [1], which provided suitable photon flux within the energy ranges of 10-100 keV. A Compton spectrometer, a backscattering instrument for inelastic scattering with high energy resolution, and an angiography station shared the beamline. The latest and largest study of intravenous coronary angiography, using a fixed protocol and involving $230 \mathrm{pa}-$ tients, completed this work [2]. Subsequently, the beamline was modified and used for high-pressure studies with a large-volume press as well as for diffraction and tomography studies of engineering materials. In 1992, DORIS was extended by modifying one of its long straight sections to a slight bend, which allowed for installing seven insertion devices. A year later, the particle physics program at DORIS ended, and it became a fully dedicated second-generation synchrotron radiation facility. One of the new bypass wiggler beamlines (BW5) was optimized for the use of high-energy synchrotron radiation around $100 \mathrm{keV}$ and equipped with a triple-axis diffractometer [3]. The instrument was successfully applied to measure structure factors in liquids and amorphous materials, to collect full data sets of highly accurate structure factors for charge-density work, to measure the spin component of the groundstate magnetization in transition-metal and rare-earth compounds, to study the diffuse scattering from stacking faults and dislocation loops in Si single crystals, and for the investigation of various aspects of structural phase transitions. A crossed-beam technique with conical slits allowed for local studies of texture, internal strain, and phase changes in gauge volumes in the bulk of materials [4].

Starting in 1992, the storage ring PETRA served as injector for the large electron/positron-proton collider HERA for particle physics research accelerating electrons or positrons up to energies of $14 \mathrm{GeV}$. Between injections, PETRA was operated at an electron energy of $12 \mathrm{GeV}$ with an emittance of $\varepsilon_{\mathrm{h}}=56 \mathrm{~nm}$.rad in the horizontal and $\varepsilon_{\mathrm{v}}=1.1 \mathrm{~nm}$. rad in the vertical direction. A 4-m-long undulator with a keyholeshaped vacuum chamber delivered X-rays for two diffraction beamlines with a brilliance of about $10^{17}$ photons $/\left(\mathrm{sec} \cdot \mathrm{mrad}^{2} \cdot \mathrm{mm}^{2} \cdot 0.1 \%\right.$
BW) at $100 \mathrm{keV}$. With one of the first series of experiments, the origin of the sharp component of the critical scattering observed with X-rays, in addition to the broad component studied with neutrons above the cubic-to-tetragonal phase transition of $\mathrm{SrTiO}_{3}$, could be clarified. On the same sample, which had been used for fundamental neutron studies, a high-resolution depth-dependent study related the sharp component to a strain gradient in the surface near-region [5].

In June 2007 HERA was closed, and PETRA was converted to the hard X-ray synchrotron radiation facility PETRA III, providing, until recently, the worldwide smallest emittance values of $\varepsilon_{\mathrm{h}}=1.2 \mathrm{~nm}$.rad and $\varepsilon_{\mathrm{v}}=12 \mathrm{pm}$.rad at $6 \mathrm{GeV}$ electron energy and photon beams of highest brilliance. One octant of the $2.3-\mathrm{km}$-long ring was partly dismantled to give room for 15 undulator beamlines at nine sectors. The first monochromatic beam was observed in July 2009 and, in 2013, all 15 beamlines were fully operational for users. In October 2012, the operation of DORIS III came to an end, and highly demanded experimental techniques were allocated in two extensions of PETRA III, each providing three additional sectors with five beamlines (see Figure 1). After more than one year of construction work for both extensions, PETRA III restarted operation in spring 2015 . Today, 23 beamlines with 57 experimental stations are available for users. Among these, there are five dedicated high-energy beamlines with 11 end stations (see Table 1).

PETRA III is one of the few facilities offering beam focusing down to a couple of micrometers at $100 \mathrm{keV}$ photon energy as a standard option at several beamlines. In this way, unique experimental capabilities for all small-beam applications were established. Furthermore, special interest is on total scattering and pair distribution function analysis to study the local atomic structure of solids, liquids, and nanomaterials. While this technique benefits from the high flux at high photon energies in general, the viable small beam option advances its applicability to ultrathin films, as illustrated in the following. Scientific activities include research on engineering materials (e.g., alloy and process development, joining techniques, fatigue properties), condensed matter physics (e.g., electronic order phenomena, thin layer growth, structure solution, and phase diagrams), chemistry (e.g., catalysis, synthesis of nanomaterials, 


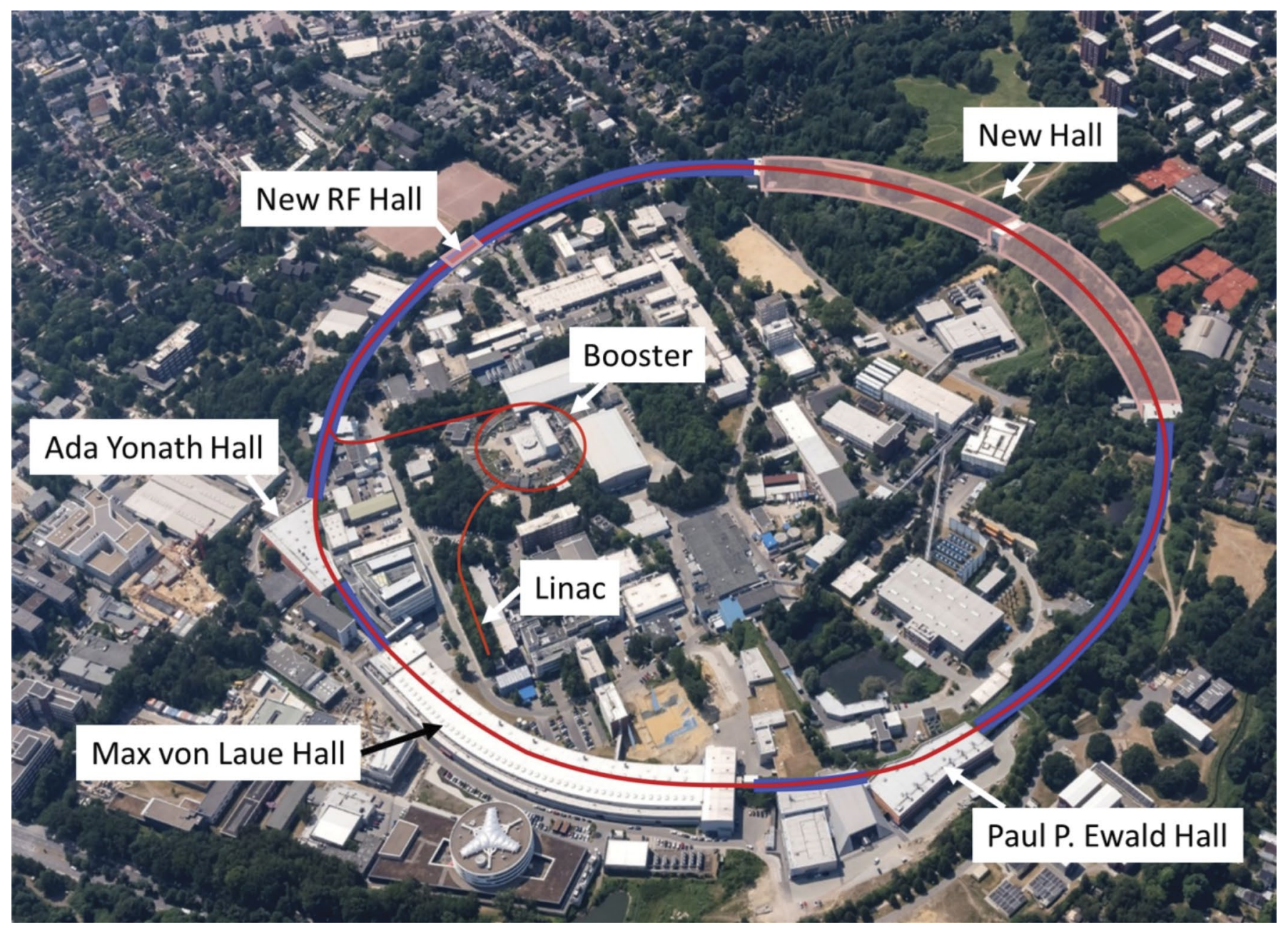

Figure 1: PETRA III on the DESY site, with its three experimental halls named after Max von Laue, Paul P. Ewald, and Ada Yonath. The figure also shows the new experimental hall and the RF hall planned for the upgrade project PETRA IV [6].

batteries, and sustainable energy sources), and geoscience (extreme conditions). Prominent examples of high-energy synchrotron radiation research at PETRA III are outlined in the following sections.

\section{Structural studies of high- $T_{\mathrm{c}}$ superconductors}

High-energy X-rays have been instrumental for studies of structural ordering phenomena in copper-oxide-based high- $T_{\mathrm{c}}$ superconductors that are found in the form of stripes or charge density waves (CDW). With their large penetration depth, the high-energy photons probe the average of the inherently inhomogeneous samples with high momentum resolution. The small scattering angles also allow us to utilize this technique for diffraction studies under high magnetic fields $\leq 17 \mathrm{~T}$, generated by a solenoid magnet [13]. Still, a large number of reflections can be accessed to survey reciprocal space up to high indexed peaks like $(8,0,0)$. The high magnetic fields have been used to study the interplay of the so-called ferro-CDW and antiferro-CDW with superconductivity at beamline P07.
In the prototypical high- $T_{\mathrm{c}}$ superconductor $\mathrm{YBa}_{2} \mathrm{Cu}_{3} \mathrm{O}_{6.67}$, CDWs appear as incommensurate lattice modulations along the crystallographic $a$ - or $b$-direction [14, 15]. While the correlation of the CDW modulation within the $\mathrm{CuO}_{2}$ plane is high, the interplane correlation is weak. This is determined from the diffraction signal that appears as a scattering rod, sharp in-plane and very broad along the $c$-axis. Maxima of the scattering rods are found at half-integer positions, which are indicative of an antiphase relation between the $\mathrm{CDW}$ modulations in adjacent $\mathrm{CuO}_{2}$ layers (see Figure 2a-c). Recently, a new variant of CDW order was found: the ferro-CDW order [16]. It exhibits a sharp peak, also along the $c$-axis at integer values of the reciprocal lattice. In this case, the CDW modulations are stacked with an in-phase relation in adjacent copper oxide layers.

The phase stability of anti-ferro-CDW, ferro-CDW, and superconductivity can be tuned with a magnetic field. At zero field, antiferro-CDW is found below the ordering temperature of about $160 \mathrm{~K}$. The intensity of the anti-ferro-CDW peak is independent of magnetic 


\section{TECHNICAL REPORTS}

Table 1: Dedicated high-energy beamlines at PETRA III (experimental hutches in parentheses).

\begin{tabular}{|c|c|c|c|}
\hline Beamline & Insertion device & Energy range & Techniques \\
\hline $\begin{array}{l}\text { P02.1 } \\
\text { P02.2 }\end{array}$ & $2 \mathrm{~m}$ undulator & $\begin{array}{c}60 \mathrm{keV} \\
26,43,60 \mathrm{keV}\end{array}$ & $\begin{array}{l}\text { powder diffraction, total scattering [7] } \\
\text { extreme conditions [8] }\end{array}$ \\
\hline P21.1 & $2 \mathrm{~m}$ undulator & $52,85,100 \mathrm{keV}$ & single crystal diffraction, total scattering, powder diffraction (EH1) \\
\hline P21.2 & $4 \mathrm{~m}$ in vacuum undulator & $38 \ldots 150 \mathrm{keV}$ & $\begin{array}{l}\text { combinations of powder \& grain-resolved diffraction, small angle } \\
\text { scattering, imaging, surface diffraction }(\mathrm{EH} 3)[11]\end{array}$ \\
\hline $\begin{array}{l}\text { P61A } \\
\text { P61B }\end{array}$ & $\begin{array}{l}10 \times 4 \text { m damping } \\
\text { wiggler }\end{array}$ & $30 \ldots 200 \mathrm{keV}$ & $\begin{array}{l}\text { energy-dispersive diffraction (EH1, EH2), white-beam radiography } \\
(\mathrm{EH} 1, \mathrm{EH} 2)[12]\end{array}$ \\
\hline
\end{tabular}

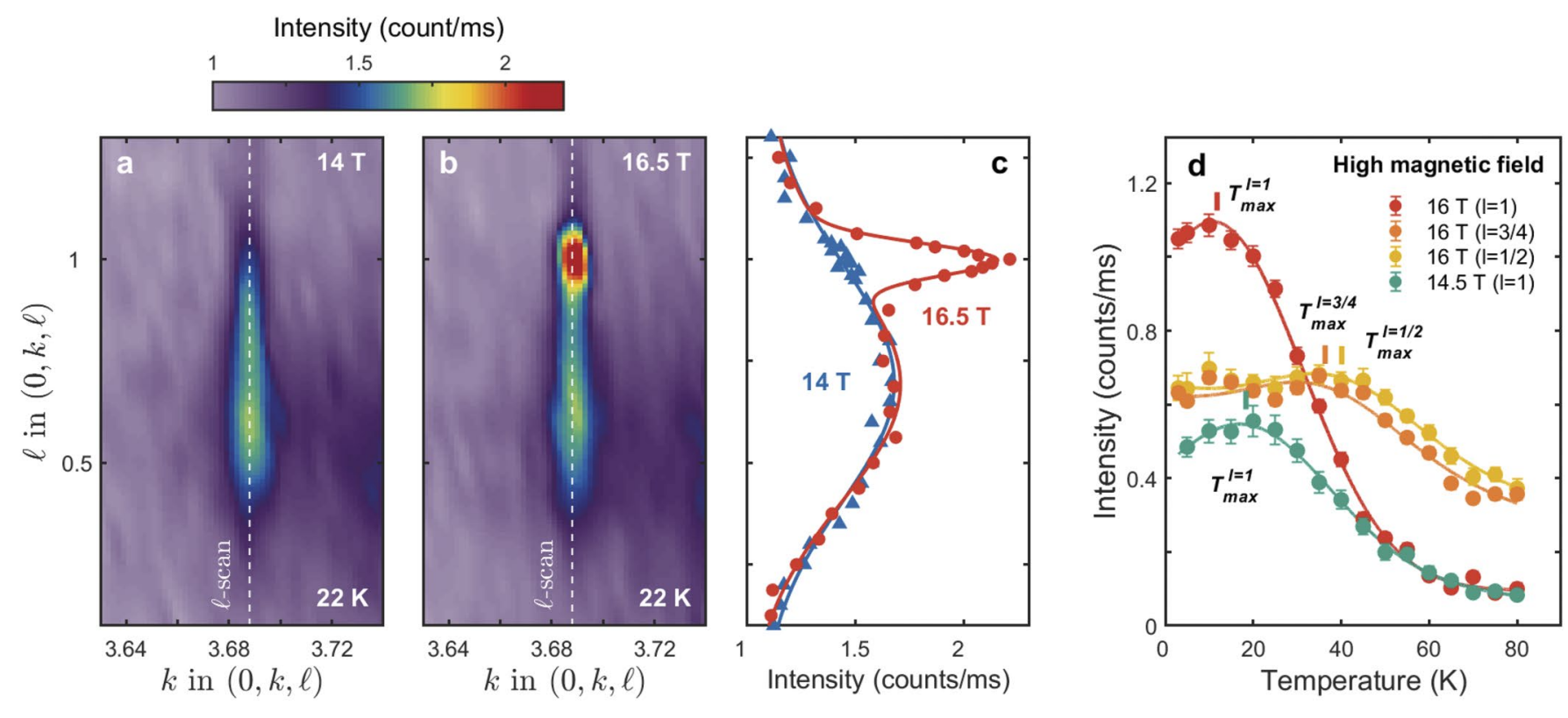

Figure 2: Field-induced emergence of a ferro-CDW on top of an anti-ferro-CDW [17]. ( $a, b)$ Diffraction intensity maps of the CDW scattering rod at fields of $B=14 \mathrm{~T}$ and $16.5 \mathrm{~T}$, respectively. The increase of intensity at the position of the ferro-CDW component at $(0,3.69,1)$ is visible. (c) Intensity profile along the vertical dashed line in $(a, b)$, probing inter-plane correlations. The highly correlated ferro-CDW is coexisting with the weakly correlated anti-ferro-CDW at $(0,3.69,0.5)$. (d) Peak intensity versus temperature at high magnetic field of the anti-ferro-CDW and ferro-CDW, reflecting the competition between superconductivity and $C D W$ order at selected values of $l$ and magnetic field. Vertical lines indicate $T_{\text {max }}$, the maximum of the temperature dependence of the charge order peak.

field for temperatures above $T_{\mathrm{c}} \sim 65 \mathrm{~K}$. The competition between superconductivity and CDW order becomes apparent from the fielddependent intensity of the charge order peak at temperatures below $T_{\mathrm{c}}$ [17]. Here, the temperature dependence exhibits a non-monotonic behavior with a maximum at the temperature $T_{\max }$. This temperature can be viewed as a characteristic temperature scale for the competition between CDW and superconductivity. For the anti-ferro-CDW,
$T_{\max }$ shifts to lower temperatures with increasing field. The extrapolation of $T_{\max }$ to $T=0$ results in a field that is close to what has been reported as $B_{\mathrm{c} 2}$, the critical field of superconductivity. The behavior of $T_{\max }$ for the ferro-CDW is different; at high field $(B>14 \mathrm{~T})$, it is always smaller than for the anti-ferro-CDW and the extrapolation to $T=0$ results in a significantly smaller critical field (see Figure $2 d)$. This implies that, in those regions of the sample that exhibit 


\section{TECHNICAL REPORTS}

ferro-CDW order, superconductivity is weaker. Such a fragile state of superconductivity has been predicted by models of pair density wave order.

\section{Real-time surface and thin film processes}

The scattering signal from thin films with thicknesses in the nanometer range is enhanced by shining the X-rays onto the surface under grazing incidence close to the critical angle of total external reflection $\alpha_{\mathrm{c}}$. At high $\mathrm{X}$-ray energies $\geq 50 \mathrm{keV}$, not only are the Bragg angles small, but also the critical angles that take values $<0.01^{\circ}$, even for high- $Z$ materials. Surface diffraction at grazing incidence around such small $\alpha_{c}$ hence requires highly precise and dedicated instrumentation to align the sample with high reproducibility and stability. Furthermore, tight focusing in the vertical to a couple of micrometers is required to keep the projected footprint on the surface short within a few millimeters. PETRA III's high brilliance up to high photon energies is key to realize stable microbeams with high flux at energies of $100 \mathrm{keV}$ and above. By combining the surface sensitivity of the grazing incidence geometry with the large range in reciprocal space captured in a single exposure on a large area detector, structural changes at surfaces, interfaces, and in thin films are traceable on the time scale of seconds. While surface diffraction has long been exploited using hard X-rays, in particular for the investigation of single-crystal surfaces and epitaxial layers, the extended panoramic overview of reciprocal space at high energies was recently applied to obtain high-quality data for a complete structural refinement of the sample system. Pioneering work on fast surface structure determination during catalytic gas reactions was reported by Gustafson et al. [18]. By rapidly turning a palladium single crystal by 90 degrees around the vertical rotation axis, they detected Bragg reflections and crystal truncation rods (CTRs) that enabled them to follow the reconstruction of the $\mathrm{Pd}(100)$ surface during carbon monoxide oxidation at a time resolution of minutes. Concurrently, the full view of reciprocal space revealed the unexpected formation of surface oxide, which would have been missed when probing only a few selected Bragg reflections with lower photon energies (see Figure 3).
More recently, total scattering in grazing incidence has successfully been applied to study the local atomic structure of amorphous and nanocrystalline (ultra)thin films with unprecedentedly low thicknesses down to a few nanometers $[19,20]$. Here, the scattering signal was collected far out in reciprocal space to $Q>20 \AA^{-1}$ on an area detector and Fourier-transformed into the atomic pair distribution function (PDF). The PDF is an ideal tool to study the short-range order of amorphous and nanocrystalline materials as well as local disorder in periodic polycrystalline structures. As a case study, we followed the evolution of strain in ultrathin platinum films during sputter-deposition in situ at a time resolution of $<1 \mathrm{~s}$ (see Figure 4). Further development allowed for resolving the local structure of the individual components of amorphous on crystalline thin film bilayer structures for next-generation electronics, such as resistive switching-based memory devices [21]. For functional thin films, the transition from the disordered state of a layer deposited at low temperature into a crystalline layer by thermal treatment often crucially affects the film properties and performance. While PDF is ideally suited to analyze the development from short- to long-range order, variable-temperature grazing incidence experiments suffer from the thermal expansion and resulting loss of sample alignment. Our current activities aim at installing a sample stabilization system that keeps the surface aligned within the tight restrictions for grazing incidence total scattering during heating and cooling.

\section{Design of engineering materials}

In the field of materials research, Helmholtz-Zentrum Geesthacht (HZG) develops lightweight construction materials for transportation applications (e.g., Mg alloys), joining technologies (e.g., laser beam welding or friction stir welding), and biomaterials (e.g., degradable alloys for implants). For the characterization of the materials and processes, HZG operates synchrotron beamlines at PETRA III in Hamburg and neutron scattering instruments at the Research Neutron Source Heinz-Maier-Leibnitz (FRM II) in Garching near Munich. This instrumentation is offered on the virtual platform GEMS (German Engineering Materials Science Centre) that serves academic and industrial users. The GEMS instruments
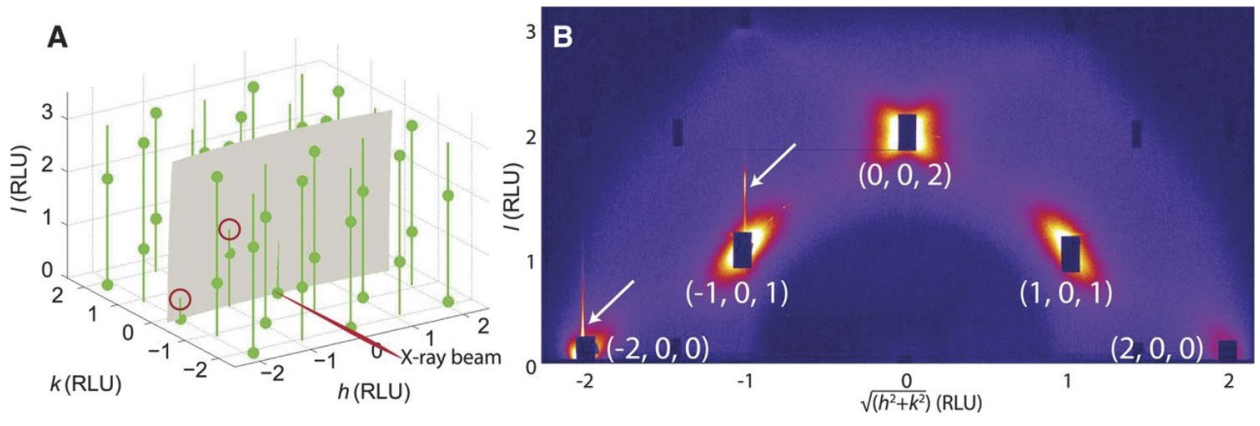

Figure 3: Example for high-energy diffraction and scattering studies on surfaces and thin films performed in the second experimental hutch of beamline P07. (A) Schematic representation of crystal truncation rods (CTRs) of a Pd(100) surface and their intersection with the Ewald sphere during rotation around the surface normal (see also movie S1 in [18]) with RLU the reciprocal lattice units. (B) Resulting image on the area detector, with the indices corresponding to the Bragg reflections of the Pd surface blocked by beam stops (dark rectangles) and the arrows indicating the positions where the CTRs cross the Ewald sphere (from [18], reprinted with permission from AAAS). 


\section{TECHNICAL REPORTS}

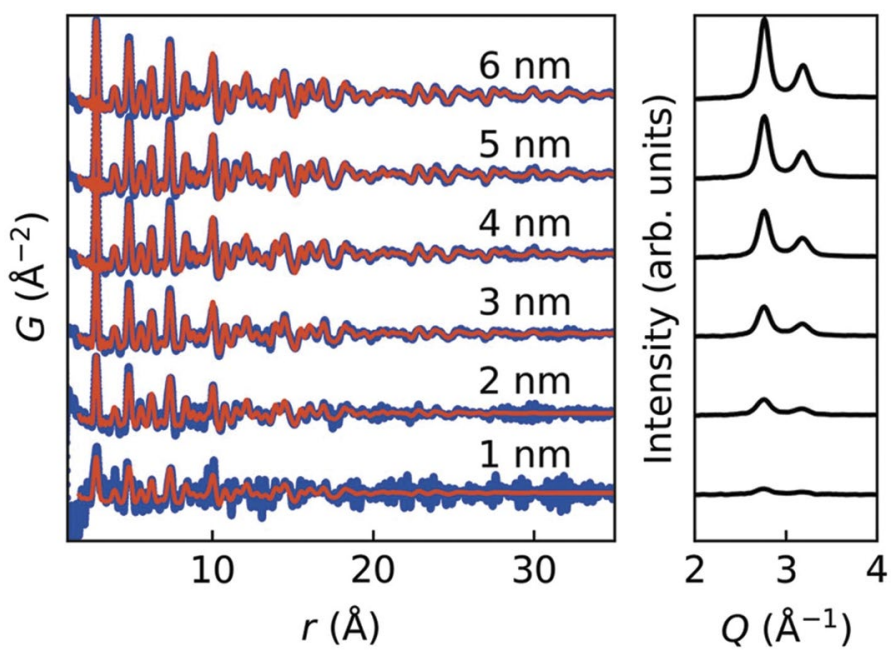

Figure 4: Pair distribution functions obtained from in situ total scattering data acquired at beamline P07-EH2 during sputtering of a Pt thin film, which demonstrate that good agreement between data (blue dots) and model (red lines) is reached at an average film thickness of $3 \mathrm{~nm}$ [19].

give HZG a direct access to diffraction and scattering, as well as imaging techniques that are essential for its research. While neutrons offer a very high penetration power and specific nuclear and magnetic contrast properties, synchrotron X-rays provide very high intensity for extreme spatial and temporal resolutions, even at energies of $100 \mathrm{keV}$. Time-resolved high-energy X-ray diffraction (HEXRD) with image rates of up to $250 \mathrm{~Hz}$ are only possible at synchrotron radiation facilities and enable in situ and operando studies of processes [22]. The diffraction beamlines operated by HZG utilize high-energy photons with both monochromatic (P07, shared with DESY [8]) and white beams (P61A), making use of the high penetration depth for the study, e.g., of large samples and industrial parts. Relevant techniques are diffraction for the analysis of phases, residual stresses and textures, and small-angle scattering for the analysis of nanostructures. Generally, an important task is the development of sample environments for in situ studies of the processes that are studied at HZG and in cooperation with external partners, based on the expertise of HZG gained over many years.

Intermetallic $\gamma$-TiAl-based alloys are interesting because of their low density of about $4 \mathrm{~g} / \mathrm{cm}^{3}$, high specific Young's modulus, and yield strength at elevated temperatures, combined with good creep properties and oxidation resistance [23]. Thus, TiAl is already being used, e.g., for aircraft turbine blades [24]. At room temperature, however, these alloys have a low tensile ductility and fracture toughness that need to be improved by tailoring the microstructure. in situ heating experiments, combining a quenching dilatometer with simultaneous HEXRD and small angle X-ray scattering (SAXS), are an ideal tool to study the formation of a second phase in metallic alloys (Figure 5). In this case, discshaped particles with a mean diameter of $12 \mathrm{~nm}$ and a mean thickness of $4.6 \mathrm{~nm}$ are formed, as can be seen in the atom probe tomography (APT) reconstruction. Only the high photon energy $(100 \mathrm{keV})$ allows using several millimeter-thick samples that are needed because of the large grain size. At this energy, the available flux enables a time resolution for SAXS of about $1 \mathrm{~s}$. The diffraction signal, which can be recorded with a second detector at the same time, provides a significantly higher time resolution $(<100 \mathrm{~ms})$. The goal of future studies is to contribute to the development of improved high-temperature materials that contribute to the reduction of fuel consumption and $\mathrm{CO}_{2}$ emission.

\section{New and emerging opportunities}

Complementing the monochromatic high-energy beamlines, the Damping Wiggler Beamline P61 in the Paul P. Ewald experimental hall constitutes the only white-beam facility at PETRA III. Here, the damping wigglers installed to achieve the low emittance of the electron beam serve as an X-ray source [25]. The white beam offers unique experimental possibilities complementary to the monochromatic beams. P61 has two experimental stations: P61A, operated by Helmholtz-Zentrum Geesthacht, for high-energy engineering materials science, and P61B, supported by DESY. When P61A is in full operation in 2021, it will provide opportunities, e.g., for near surface
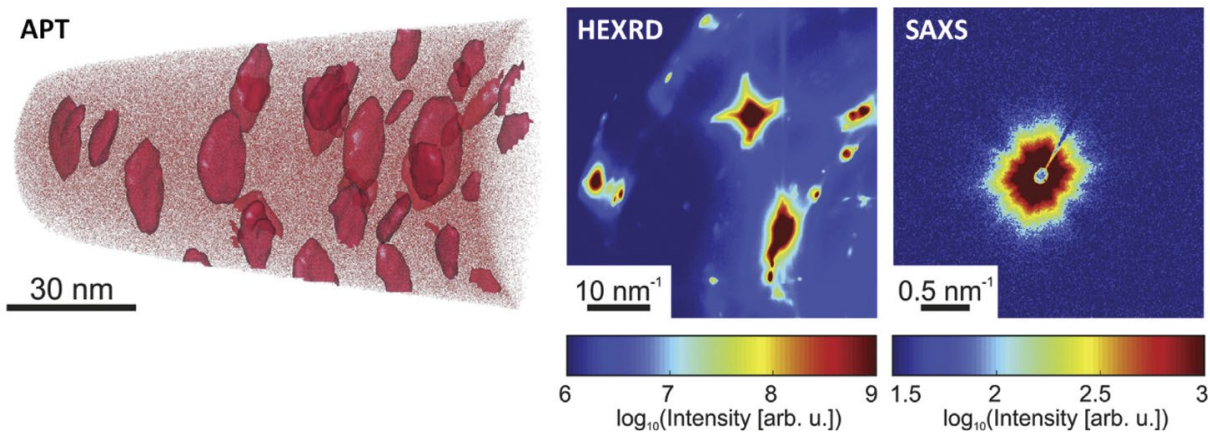

Figure 5: Ti-44Al-7Mo (at.\%) specimen heated at a constant rate of $10 \mathrm{~K} / \mathrm{min}$ to $680{ }^{\circ} \mathrm{C}$. In the atom probe tomography (APT) image, Mo/TiO isoconcentration surfaces allow us to visualize the disc-shaped $\gamma$ precipitates in the ordered $\beta_{o}$ matrix. The corresponding HEXRD and HESAXS images were obtained from an in situ heating experiment at a temperature of $680^{\circ} \mathrm{C}$. The XRD image shows diffraction spots from large matrix crystallites, while the SAXS signal gives the size of precipitates and is also sensitive to very small precipitate clusters with sizes below a few nanometers that cannot be seen by XRD (reprinted from [23] with permission from Elsevier). 


\section{TECHNICAL REPORTS}

and bulk residual stress, texture and phase analysis, making use of a gauge volume determined by crossed beams defined by the entrance and exit slits. In addition, Laue diffraction methods and high-speed imaging will be offered. P61B will be dedicated to a large volume press (LVP, see Figure 6) to expand DESY's extreme conditions research facilities and complement the diamond anvil cells driven program by the Extreme Conditions Beamline P02.2 [8]. The LVP is the first multi-anvil device, using six hydraulic rams operated at a synchrotron light source producing loads of up to 1500 tons [12]. This translates to isotropic pressures on large cylindrical samples of up to $4 \mathrm{GPa}$ for $1 \mathrm{~cm}$ sample length and up to $100 \mathrm{GPa}$ for a length of $0.5 \mathrm{~mm}$. Anisotropic compression and resistive heating up to $2000{ }^{\circ} \mathrm{C}$ broaden the available methods for earth science, materials synthesis, and deformation at PETRA III. Energy-dispersive X-ray diffraction using a part of the wiggler spectrum enables probing the structure and properties of materials in situ under high pressures and high temperatures at a time resolution of 1-10 s. Until completion of the instrument in 2021, the LVP is available to users in a temporary setup and for offline studies without X-rays.

The Swedish Materials Science Beamline P21 [11] in the Ada Yonath extension hall was designed according to the requirements of the Swedish synchrotron user community as the high-energy complement to X-ray beamlines at MAX IV in Lund, Sweden, which operate at photon energies up to $40 \mathrm{keV}$. With a total length of almost $150 \mathrm{~m}$, the inline branch P21.2 offers beam sizes, between $2 \times 6 \mathrm{~mm}^{2}$ and $1 \times 5 \mu \mathrm{m}^{2}$, of low energy bandwidth in the energy range $40-150 \mathrm{keV}$. Besides surface diffraction, P21.2 focuses on multi-dimensional imaging techniques like grain mapping (from near to very far field), microbeam computed to- mography $(\mu \mathrm{CT})$ with absorption and phase contrast, as well as small and wide angle scattering (SAXS/WAXS) to describe structure on the length scale from angstroms to millimetres. In addition, the canted beamline P21.1 is dedicated to the study of structural ordering, ranging from single crystals to liquids by analysis of diffuse scattering up to $100 \mathrm{keV}$, thus complementing the capabilities of the Powder Diffraction and Total Scattering Beamline P02.1 operated at $60 \mathrm{keV}$ [7]. Though only sector P21 is funded by the Swedish government, Swedish users can, up to an equivalent share, access all other beamlines as well, rendering P21 partly available to general users. P21 has started user operation in the second half of 2019 and is currently ramping up to its full potential.

Worldwide, the next wave of progress in X-ray science will be stimulated by the availability of storage ring facilities with much lower emittance getting closer and closer to the diffraction limit even for hard X-rays. This became possible by using a novel multibend achromat lattice, pioneered at MAX IV. Almost all hard Xray facilities perform or plan upgrades. DESY currently plans an upgrade of PETRA III, realizing a hybrid seven-bend achromat lattice based on ESRF-EBS design in the existing tunnel of $2304 \mathrm{~m}$ circumference, and aims for emittance values of $\varepsilon_{\mathrm{h}}=20 \mathrm{pm} . \mathrm{rad}$ and $\varepsilon_{\mathrm{v}}=4$ pm.rad at $6 \mathrm{GeV}$ electron energy. The new ultralow-emittance source project PETRA IV [6] is expected to provide almost diffraction-limited beams at $10 \mathrm{keV}$ and a brilliance at $100 \mathrm{keV}$, which is as high as obtained today at $10 \mathrm{keV}$. Figure 7 shows the enormous projected brilliance gains, especially in the spectral range of high-energy synchrotron radiation. One major advantage of the new source will be the possibility to focus the X-rays into smaller spot sizes than to date, with similar dimensions in the vertical and
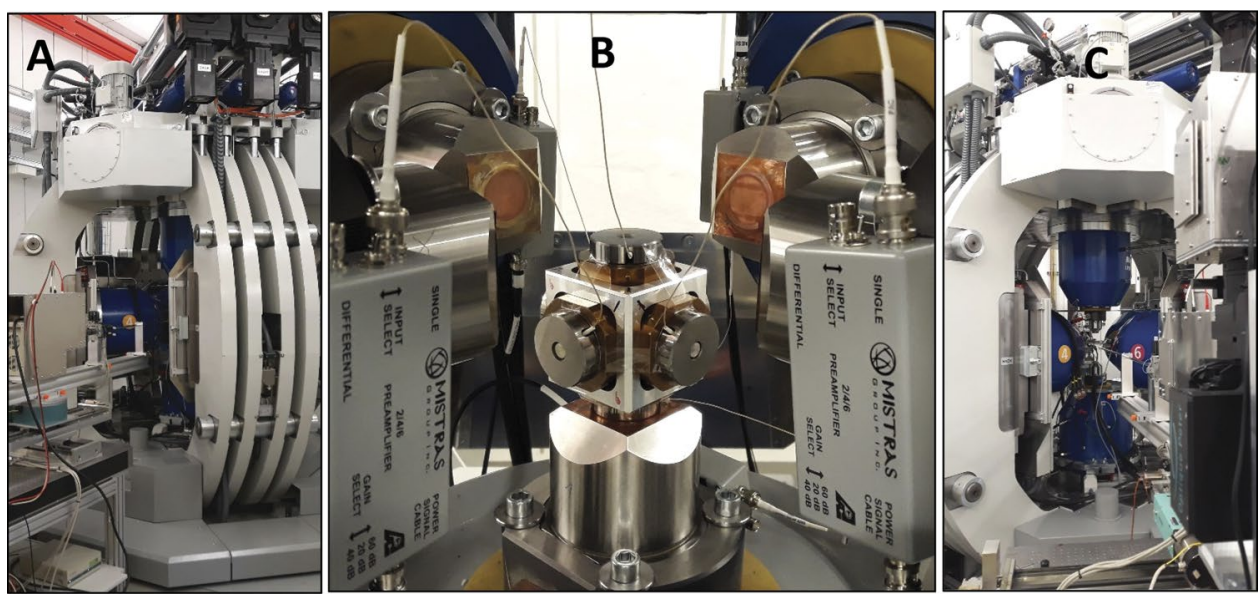

Figure 6: (A and C) The $15 \mathrm{MN}$ large volume press with six independently controlled hydraulic rams at the Damping Wiggler Beamline P61 [12], which allows for heating to over $2000{ }^{\circ} \mathrm{C}$ on samples in assemblies at high pressures up to $30 \mathrm{GPa}$, the routine maximum pressure. Energy-dispersive X-ray diffraction is achieved using a high-purity Ge-detector with collimator-slit setup. Radiography imaging is achieved using a thin scintillator and an X-ray microscope. Cumulative power of the white X-ray beam in $1 \times 1 \mathrm{~mm}^{2}$ at $40 \mathrm{~m}$ from the 10 wigglers is $121 \mathrm{~W}$ [25], and $12 \mathrm{~W} / \mathrm{mm}^{2}$ at the LVP (at $136 \mathrm{~m}$ ) after heat load filters. Max. photon flux at $50 \mathrm{keV}$ in a $1 \times 1 \mathrm{~mm}^{2}$ aperture at the LVP is $10^{12} \mathrm{ph} / \mathrm{s} / 0.1 \% \mathrm{~B}$. W., with flux approaching $10^{10}$ ph/s/0.1\% b.w. beyond $200 \mathrm{keV}$. For more details, see https://tiny.cc/petra3p61/. (B) Example of an assembly of six anvils inside the LVP, ready to compress a cube with a rock specimen inside. Here, each anvil has a piezoelectric sensor mounted on its back to record and locate micro-cracking in the sample under stress at high pressure and high temperature (temperature is generated by electrical resistive heating of an internal furnace). In the future, X-ray imaging will be used to visualize cracking and quantify sample strain; X-ray diffraction will be used to estimate stress. 


\section{TECHNICAL REPORTS}

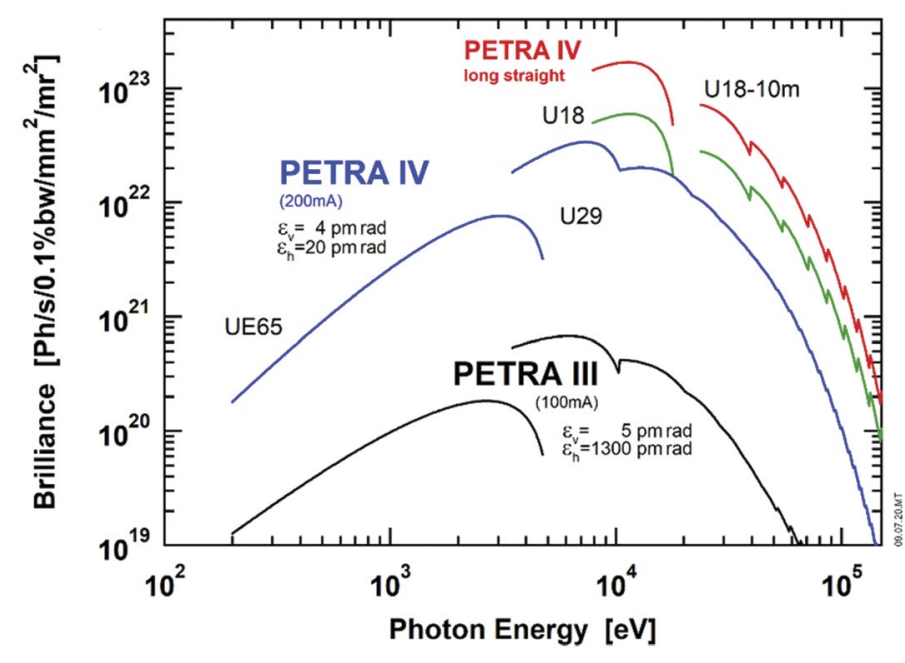

Figure 7: Spectral brilliance of PETRA III for a ring current of $100 \mathrm{~mA}$ compared with the expected performance of PETRA IV in high-brilliancel high-coherence mode of operation for a ring current of $200 \mathrm{~mA}$ [6]. For the soft X-ray spectrum, the comparison is made for a $5 \mathrm{~m}$ UE65 undulator currently installed at beamline P04 at PETRA III. In the hard/high-energy spectral range, the comparison is made for a $5 \mathrm{~m}$ U29 undulator installed at P10. In the high-energy X-ray range, significant gains in brilliance can be achieved with a U18 undulator; i.e., a $5 \mathrm{~m}$ device in a regular ID section (green curve) and $10 \mathrm{~m}$ device in one of the four long straight sections with optimized electron beam parameters (calculated by Markus Tischer, DESY).

the horizontal. Along with surface diffraction, grain mapping, and other spatially scanning techniques, diffraction-based computed tomography (CT) methods will largely benefit from the voxel resolution on the scale of a few cubic micrometers. In this way, phase information in reciprocal space (XRD-CT) and real space (PDFCT) of large objects with crystalline and/or amorphous components will be retrieved in unprecedented detail. Future advanced optics, such as multi-layer Laue lenses for high photon energies and high- $Z$ area detectors with kilohertz readout and small pixels, will pave the way to coherence applications using high-energy X-rays. Their potential for the fields of dynamics and nanoscale $\mathrm{X}$-ray microscopy will be explored by using techniques like X-ray photon correlation spectroscopy (XPCS), Bragg coherent diffractive imaging (CDI), and ptychography with high-energy X-rays.

\section{References}

1. W. Graeff et al., Rev. Sci. Instrum. 60, 1457-1459 (1998). doi:10.1063/1.1140962

2. W. R. Dix et al., J. Synchrotron Radiat. 10, 219-227 (2003). doi:10.1107/ S0909049503001973

3. R. Bouchard et al., J. Synchrotron Radiat. 5, 90-101 (1998). doi:10.1107/ S090904959701457X

4. H. F. Poulsen et al., J. Synchrotron Radiat. 4, 147-154 (1997). doi:10.1107/ S0909049597002021

5. H. Hünnefeld et al., Phys. Rev. B - Condens. Matter Mater. Phys. 66, 141131-1411314 (2002)

6. C. Schroer et al., DESY, doi:10.3204/PUBDB-2019-03613(2019).

7. A.-C. Dippel et al., J. Synchrotron Radiat. 22, 675-687 (2015). doi:10.1107/ S1600577515002222
8. H.-P. Liermann et al., J. Synchrotron Radiat. 22, 908-924 (2015) doi:10.1107/S1600577515005937

9. N. Schell et al., Mater. Sci. Forum 772, 57-61 (2013). doi:10.4028/www. scientific.net/MSF.772.57

10. F. Bertram et al., AIP Conf. Proc. 1741, 040003-1-040003-4 (2016).

11. Z. Hegedüs et al., IOP Conf. Ser. Mater. Sci. Eng. 580, 012032(2019).

12. S. Bhat et al., Chem. 26, 2187-2194 (2020). doi:10.1002/chem.201904529

13. A. T. Holmes et al., Rev. Sci. Instrum. 83, 023904-1-023904-6 (2012). doi: $10.1063 / 1.3688657$

14. G. Ghiringhelli et al., Science 337, 821-825 (2012). doi:10.1126/science. 1223532

15. J. Chang et al., Nature Phys. 8, 871-876 (2012). doi:10.1038/nphys 2456

16. S. Gerber et al., Science 350, 949-952 (2015). doi:10.1126/science.aac6257

17. J. Choi et al., Nature Commun. 11, 1-8 (2020).

18. J. Gustafson et al., Science 343, 758-762 (2014). doi:10.1126/science .1246834

19. A.-C. Dippel et al., IUCrJ 6, 290-298 (2019). doi:10.1107/ S2052252519000514

20. M. Roelsgaard et al., IUCrJ 6, 299-304 (2019). doi:10.1107/ S2052252519001192

21. A.-C. Dippel et al., Nanoscale 12, 13103-13112 (2020). doi:10.1039/ D0NR01847C

22. P. Staron et al., Adv. Eng. Mater. 13, 658-663 (2011). doi:10.1002/ adem. 201000297

23. P. Erdely et al., Acta Mater. 164, 110-121 (2019). doi:10.1016/j.actamat.2018.10.042

24. B. P. Bewlay et al., MRS Proc. 1516, 49-58 (2013). doi:10.1557/opl.2013.44 25. W. Drube et al., AIP Conf. Proc. 1741, 020035-1-020035-4 (2016)

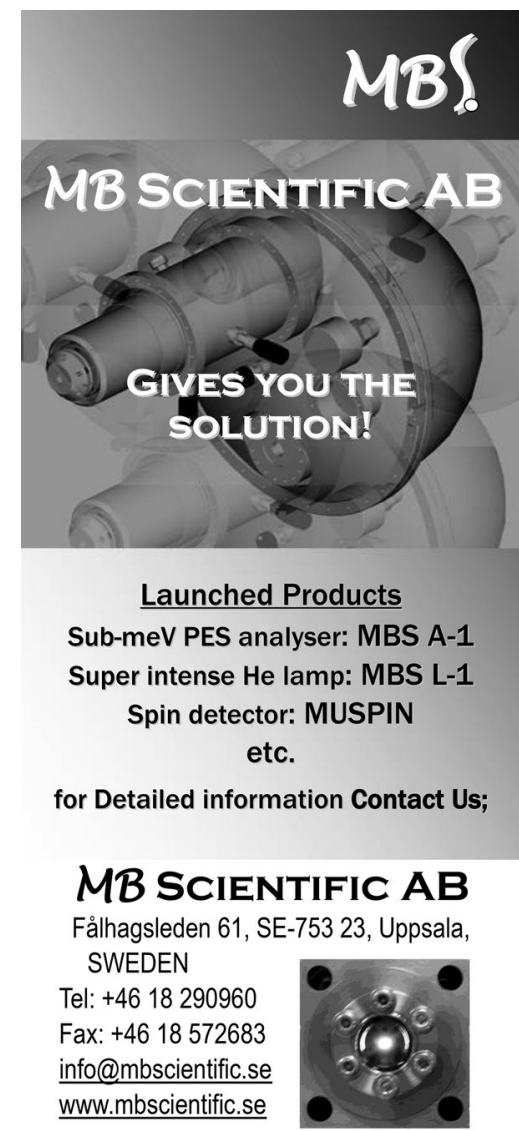

